\title{
Methods of Spectral Analysis of Traction Voltages and Currents as Random Functions
}

\author{
Anatolii Nikitenko \\ Dnipropetrovsk National University of Railway Transport named after Ac. V. Lazaryan \\ Dnipropetrovsk, Ukraine \\ nikitenko.diit@gmail.com
}

\begin{abstract}
The paper shows the methods of spectral analysis of stochastic traction voltages and currents. The results of analysis are shown for the voltage, which was recorded in the process of real operation of EPL2T electric multiple-unit train.
\end{abstract}

Keywords - correlation, spectrum, voltage, train, recuperative braking.

\section{INTRODUCTION}

Nowadays almost the half of main and suburban railway transport, all mine and pit railway transport and, of course, all urban electric transport of Ukraine are exploited using the DC traction power supply system. Electric locomotives, trains, multiple-unit trains, metro cars use the DC voltage with nominal rate from 550 to $3000 \mathrm{~V}$. However, electric rolling stock (ERS) is a nonstationary, sharply changing, non-linear load, where frequent dynamic operation modes of power circuit elements give rise to flowing of stochastic electric tractive currents $I(t)$ in power circuits of ERS and power supply network $[1,2]$. Furthermore, the elements of ERS power circuits are under the significant influence of randomly time-varying voltage $U(t)$ of power supply network (Fig. 1).

\section{THEORETICAL ANALYSIS}

As for as the voltage $U(t)$ on the current collector of ERS and consumed current $I(t)$ are stochastic processes, their spectral composition should be carried out using the probabilistic methods which are based on the theory of random functions. The harmonic composition of DC voltage and current is assumed to be a probabilistic one. For their spectral composition analysis, we may consider the application of two different methods: the statistical-spectral method and the correlation-spectral one.

The first one is based on the discrete Fourier transformation applied to the voltage $U(t)$ and current $I(t)$, which are non-sinusoidal and non-periodical functions. We conduct our analysis for a general random function $f(t)$ that can be replaced by $U(t)$ or $I(t)$.

For this purpose, the continuous function $f(t)$ is discretized with the time intervals $\Delta t=t_{n+1}-t_{n}$ (Fig. 2).

In Fig. 2: $N$ is a total number of discretized intervals; $n=0,1, \ldots, N$; then $\Delta t=T / N$.

Two ways of the expansion $f(t)$ into a series are possible: by the application of the piecewise constant approximation (step curve $0,1, \ldots, 5$ in Fig. 2) and the piecewise-linear approximation of the function $f(t)$.

Application of the piecewise-constant approximation assumes that the discretized function $f(t)$ has a constant value within the range of any interval $\Delta t$. Then we split the well-known expression [3] for calculation of a complex amplitude of $k$ - th harmonic

$$
\underline{C}^{(k)}=\frac{2}{T} \int_{0}^{T} f(t) \mathrm{e}^{-j k \omega t} d t
$$

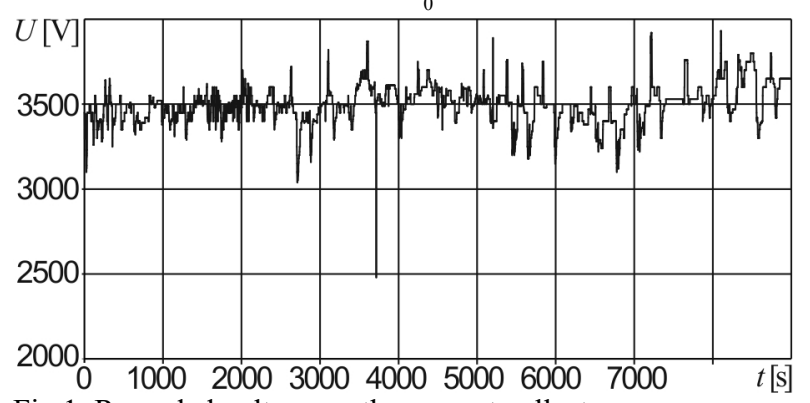

Fig.1. Recorded voltage on the current collector

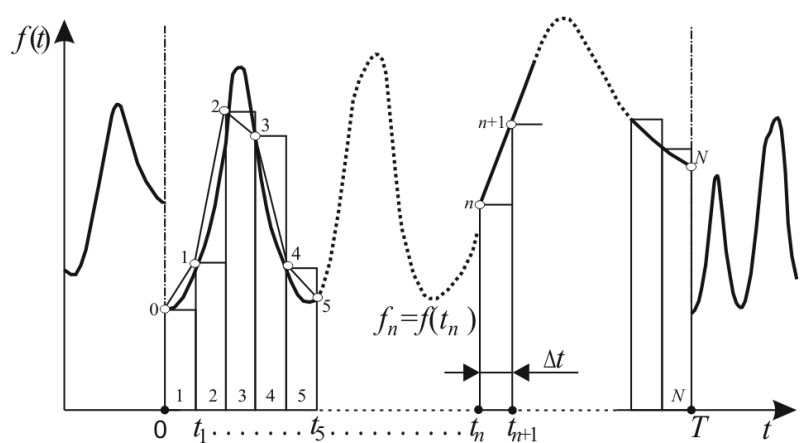

Fig.2. The discretization of characteristic of random function 
into the sum of integrals (according to Fig. 2):

$$
\begin{gathered}
\underline{C}^{(k)}=\frac{2}{T}\left[\int_{0}^{t_{1}} f\left(t_{0}\right) \mathrm{e}^{-j k \omega t} d t+\int_{t_{1}}^{t_{2}} f\left(t_{1}\right) \mathrm{e}^{-j k \omega t} d t+\ldots\right. \\
\left.\ldots+\int_{t_{n}}^{t_{n+1}} f\left(t_{n}\right) \mathrm{e}^{-j k \omega t} d t+\ldots+\int_{t_{N-1}}^{T} f\left(t_{N-1}\right) \mathrm{e}^{-j k \omega t} d t\right]= \\
=\frac{2}{T} \sum_{t_{n}=0}^{T} \int_{t_{n}}^{t_{n+1}} f\left(t_{n}\right) \mathrm{e}^{-j k \omega t} d t .
\end{gathered}
$$

Whereas the value of the function $f\left(t_{n}\right)$ is constant within the range from $t_{n}$ to $t_{n+1}$, the integrand is easy integrated and is written as

$$
\underline{C}^{(k)}=\frac{2}{T} \frac{1}{-j k \omega} \sum_{t_{n}=0}^{T} f\left(t_{n}\right)\left[\mathrm{e}^{-j k \omega t_{n+1}}-\mathrm{e}^{-j k \omega t_{n}}\right] .
$$

After taking into account that $\omega T=2 \pi$ and $t_{n}=n T / N$, we get the expression of the complex amplitude of $k$-th harmonic for the function $f(t)$ in case of its piecewise constant approximation

$$
\underline{C}^{(k)}=-\frac{1}{j \pi k} \sum_{n=1}^{N} f_{n} \mathrm{e}^{-j \frac{2 \pi k n}{N}}\left(\mathrm{e}^{-j \frac{2 \pi k}{N}}-1\right) .
$$

Application of the piecewise-linear approximation allows us to describe the function $f(t)$ within the interval $\left[t_{n}, t_{n+1}\right]$ by such linear function

$$
f(t)=\frac{t-t_{n}}{t_{n+1}-t_{n}}\left(f\left(t_{n+1}\right)-f\left(t_{n}\right)\right)+f\left(t_{n}\right),
$$

where $t \in\left[t_{n}, t_{n+1}\right]$. The function described by (5) works for each discretization interval $\Delta t$ taken separately and only within the range of the mentioned above.

If we substitute (5) in (1), we get the following

$$
\underline{C}^{(k)}=\frac{2}{T} \int_{0}^{T}\left\{\frac{t-t_{n}}{t_{n+1}-t_{n}}\left[f\left(t_{n+1}\right)-f\left(t_{n}\right)\right]+f\left(t_{n}\right)\right\} e^{-j k \omega t} d t .
$$

After that, we split (6) into the sum of integrals and integrate it to get the final formula of the complex amplitude for the case of the piecewise-linear approximation:

$$
\begin{gathered}
\underline{C}^{(k)}=-\frac{1}{j \pi k} \sum_{n=1}^{N} \mathrm{e}^{-j \frac{2 \pi k n}{N}}\left\{( f _ { n + 1 } - f _ { n } ) \left[(n+1) e^{-j \frac{2 \pi k}{N}}-n+\ldots\right.\right. \\
\left.\left.\ldots+\frac{N}{j 2 \pi k}\left(e^{-j \frac{2 \pi k n}{N}}-1\right)-n\left(e^{-j \frac{2 \pi k n}{N}}-1\right)\right]+f_{n}\left(e^{-j \frac{2 \pi k n}{N}}-1\right)\right\} \cdot(7)
\end{gathered}
$$

Subsequently, (4) or (7) can be used to get the spectral composition of the random function $f(t)$ representing voltage $U(t)$ or current $I(t)$. The expressions give virtually the same result and the difference between the results is less than $1,0 \%$.

Equations (4) and (7) describe the harmonics by the complex numbers, thus the amplitude of $k$ - th harmonic in the unknown Fourier series of the function $f(t)$ is

$$
f(t)=\sum_{k=0}^{s} A_{m}^{(k)} \sin \left(k \omega t+\psi^{(k)}\right),
$$

where $A_{m}^{(k)}=\left|\underline{C}^{(k)}\right|$ and $\psi^{(k)}=\arg \left(\underline{C}^{(k)}\right)$

This method of spectral composition defining allows us to obtain the amplitude-frequency and phasefrequency responses only for a certain $f(t)$, which constitutes a particular case of some random function of voltage or current. However, a large number of load characteristics of $U(t)$ and $I(t)$ changes drastically and has a random component. This feature complicates the calculation of accurate data for amplitudes and frequencies of harmonics discrete spectrum. Therefore, it is necessary to distinguish between the harmonics in the spectrum that are caused by random factors and those that appear in all characteristics (i.e. in the whole random process). To accomplish the task, we use for determination of the random process spectrum its correlation function, that is, we apply Fourier transformation not to the stochastic function itself, but to its correlation function. This is correlation-spectral method.

A correlation function is the most significant characteristic of a random process and characterizes its internal structure. The function provides the information about the correlation degree between the voltage (or current) values at different moments of operation.

As it is known, the correlation function of random process [2] is defined, for example, for the voltage by the expression

$$
K_{U}(\tau)=\frac{1}{T-\tau} \sum_{t=0}^{T-\tau}\left[U(t)-m_{U}\right]\left[U(t+\tau)-m_{U}\right],
$$

where $T-$ is the period of the voltage (or current); $U(t), U(t+\tau)$ are the values of random function at the moments $t$ and $(t+\tau)$, respectively; $m_{U}-$ is the mathematical expectation of random function.

It is known [4] that correlation functions of random processes of voltage and current in sharply changing loads are not attenuate in the course of $\tau$ and this fact indicates a non-ergodic random process. The sustained part of the correlation function (frequently called the "tail" of correlation function) has the same frequencies as the random process [5]. In connection with this, Fourier transformation has to be applied to the "tail" of 
correlation function for the analysis of spectral composition of periodical components of the voltage and current. This method allows us to differentiate the periodical components from the ergodic random process which is impossible in case of application of Fourier transformation to the random process as a whole. One of the following formulas of correlation functions can characterize the beforementioned ergodic random process:

$$
\begin{gathered}
K_{U}(\tau)=D \mathrm{e}^{-\alpha|t|} \\
K_{U}(\tau)=D \mathrm{e}^{-\alpha|t|} \cos \omega_{0} \tau \\
K_{U}(\tau)=D \mathrm{e}^{-\alpha|t|}\left(\cos \omega_{0} \tau+\frac{\alpha}{\omega_{0}} \sin \omega_{0}|\tau|\right),
\end{gathered}
$$

where $D-$ is a dispersion of random process; $\alpha-$ is the attenuation coefficient of the correlation function; $\omega_{0}-$ is the eigenfrequency of the correlation function.

So we represent the voltage (or traction current) as the sum of random function $U^{\prime}(t)$ with an attenuating correlation function described by any of the expressions given by (10), (11), (12) and low-frequency periodical components:

$$
U(t)=U^{\prime}(t)+\sum_{k=1}^{n} U_{m}^{(k)} \sin \left(k \omega t+\psi^{(k)}\right),
$$

where $U_{m}^{(k)} \quad$ - is the constant amplitude of $k$-th periodical component of the voltage across ERS current collector; $k \omega-$ is the frequencies of $k$-th periodical component; $\psi^{(k)}-$ is its initial phases.

As a result, we can extract the ergodic random function $U^{\prime}(t)$ from the non-ergodic random process $U(t)$ (13) and define what periodical oscillations (their amplitudes or frequencies) are present in the random process spectrum.

If the interval of registration $T$ of random function is much more than period $2 \pi / k \omega$ of the lowest frequency component, (13) can be represented as

$$
K_{U}(\tau)=K_{U}^{\prime}(\tau)+\sum_{k=1}^{n} \frac{U_{m}^{(k) 2}}{2} \cos k \omega \tau
$$

where $K_{U}{ }^{\prime}(\tau) \quad-$ is any of the correlation functions described by (10), (11) and (12).

Therefore, if the sinusoidal components are present in the random function describing the voltage across the current collector or the tractive current, the "tail" of the correlation function is the sum of cosines of the same frequencies, where cosine amplitude is equal to the half of square amplitude of corresponding sinusoidal component of the voltage or current characteristics.

\section{ANALYSIS OF RESULTS}

For the experimental analysis, voltage and current characteristics have been recorded in the process of real operation of VL8 and VL11M6 locomotives, EPL2T multiple-unit train in the sections of Lviv and Prydniprovsk railways and T4D tram. The example of characteristic of the voltage across the current collector for EPL2T electric multiple-unit train is shown in Fig. 1. All records have been analyzed using the methods mentioned above for the recuperative braking modes [1] and the results of voltage analysis are shown in Figs. 3, 4 and 5 for EPL2T electric multiple-unit train.

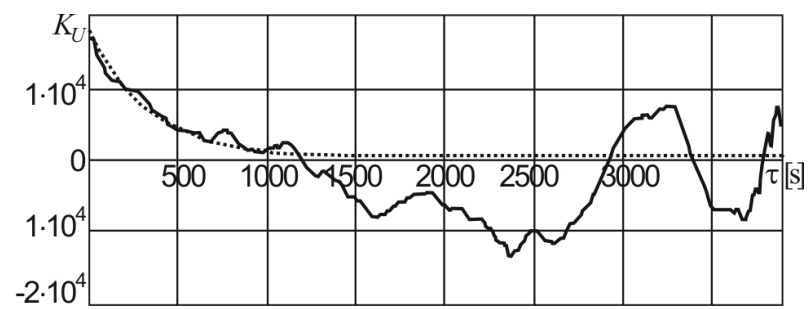

Fig.3. Correlation function of the voltage

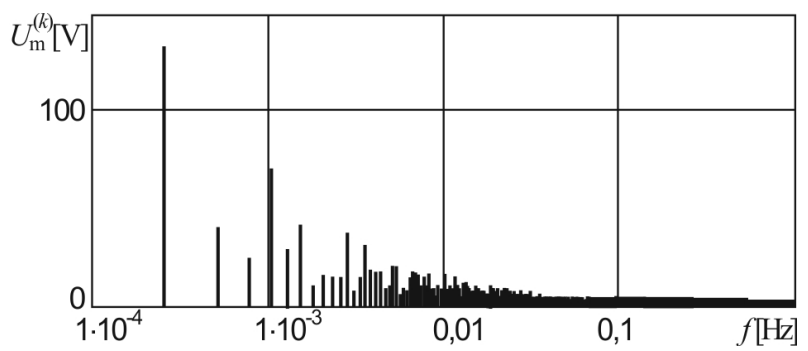

Fig.4. Spectrum of the instant voltage function

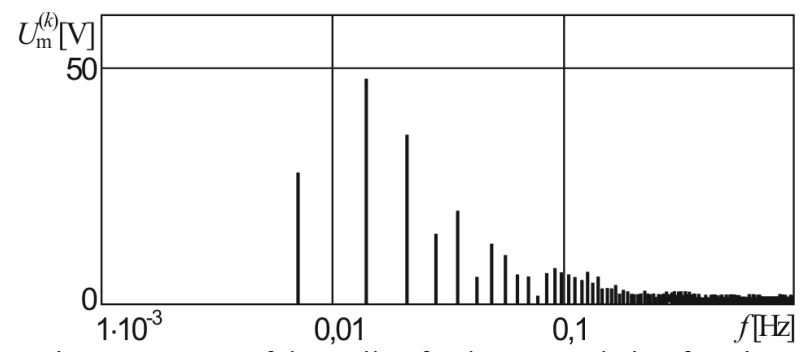

Fig.5. Spectrum of the "tail" of voltage correlation function

The shape of correlation functions shows that the voltage characteristic has the low-frequency periodical components (interharmonics). Spectrum of the "tail" of correlation function is thinned in comparison with spectrum for the instant voltage function. 


\section{CONCLUSIONS}

1. Voltage and current of DC ERS are the stationary non-ergodic random functions in recuperative braking mode.

2. The oscillations of current are random values and are changing in the wide range in the recuperative braking mode. These oscillations depend on the ERS type and change drastically in trams. The current values are frequently drifted in the region of its lowest values.

3. The sign changing in the "tail" of correlation function show that characteristics have interharmonics.

4. The amplitude spectrum of instant values $U(t)$, $I(t)$, and, of course, the correlation function spectrum are induced by the components with the frequency range $0,001 \ldots 1 \mathrm{~Hz}$. The current of trams has higher frequencies than electric locomotives and trains.

\section{REFERENCES}

[1] A. V. Nikitenko and M. O. Kostin, "Statistic, probabilistic, correlation and spectral analyses of regenerative braking current of DC electric rolling stock," in Science and Transport Progress. Bulletin of Dnipropetrovsk National University of Railway Transport, vol. 3(51), Dnipropetrovsk, Ukraine: Publ. dep. of DIIT, 2014, pp. 51-64.

[2] O. I. Sablin, "Improvement of the Consuming Efficiency of DC Electric Rolling Stock," Ph.D. dissertation, Dept. Electric Transport, Dnipropetrovsk National University of Railway Transport, Dnipropetrovsk, Ukraine, 2009.

[3] M. O. Kostin and O. H. Sheikina, Theory of Electrical Engineering, vol. 1, Dnipropetrovsk, Ukraine: Publ. dep. of DIIT, 2006, 336 p.

[4] I. V. Zhezhelenko and Yu. L. Sayenko, Eds., "Spectrum analysis of a load current of the interharmonic sources in industrial electrical networks," in Bulletin of Pryazovskyi State Technical University, vol. 12, Mariupol, Ukraine: Publ. dep. of PSTU, 2002, pp. 194-201.

[5] I. V. Pugachov, Introduction to Probability Theory, Moscow, USSR: Science, 1968, 364 p.

http://ieeexplore.ieee.org/xpl/articleDetails.jsp?arnumbe $\underline{\mathrm{r}=73333588 \& \text { content } T y p e=\text { Conference }+ \text { Publications }}$ 